\title{
BEM and FEM simulations of the vibro- acoustical response of finite noise barriers
}

\author{
S. Gasparoni, M. Ralbovsky, M. Haider, R. Wehr \& M. Conter \\ AIT Austrian Institute of Technology, Austria
}

\begin{abstract}
Noise barriers are one of the primary means in noise abatement for traffic-related noise in the proximity of roads and railways. The efficiency of noise barriers concerning sound absorption and sound insulation nowadays is evaluated with different experimental methods. On the other hand, simulations are very helpful for noise barrier manufacturers and are a cost-efficient tool for predicting and planning new noise barrier solutions.

In the following paper, the focus is placed on the vibro-acoustical interactions of noise barriers with the surrounding sound field. This means that the sound produces a vibration in the barrier which then emits additional sound waves. This aspect, often ignored, is responsible for the transmission of sound through the noise barrier. Boundary and finite elements methods are best suited to simulate this kind of interaction. Simulations have been performed and different sound sources (plane wave, point source, line source) have been analysed.
\end{abstract}

Keywords: noise barriers, noise abatement, diffraction, 3D-BEM simulations.

\section{Introduction}

Noise barriers together with other noise abatement tools are increasing with the increase of transportation traffic.

Usually attention is focussed on the noise diffracted by the barriers, which can be simulated with Boundary Element methods [1,2] and can be measured using the Adrienne method [3, 4].

Another feature that characterizes the barrier is the transmission, which is the noise transmitted by the barrier itself, without taking into account the noise diffracted. In order to measure this, the Adrienne method is used and a combination of finite elements methods and boundary elements methods is used, as the first analyses the vibration of the barrier and the second examines the 
propagation in space of the secondary sound waves. We are performing threedimensional simulations with LMS Virtuallab. The vibrational FEM model has been performed with Ansys.

\section{Investigation}

In our investigation, LMS virtual lab has been used. This software performs BEM and FEM simulations in a three-dimensional space. Using $3 \mathrm{~d}$ software instead of $2 \mathrm{~d}$ imposes the use of a finite noise barrier (an element of a barrier). Our noise barrier element is $5 \mathrm{~m}$ wide, $4 \mathrm{~m}$ high and $10 \mathrm{~cm}$ deep. The material used is concrete with $\mathrm{E}=30 \mathrm{GPa}, v=0.18, \rho=2500 \mathrm{~kg} / \mathrm{m}^{3}$.

The LMS software would allow an overall analysis starting from the sound source, letting the sound propagate until the noise barrier, applying the sound pressure on the barrier, analysing the produced vibrations and finally calculating the sound derived from the barrier vibration. Unluckily, being the barrier finite in dimension, there would be a big amount of sound propagating from the source and being diffracted by the sides of the barrier impinging on the receiver. This would completely hide the contribution of secondary sound.

In order to avoid this problem, we do not simulate the source; instead, we simulate the noise barrier vibration caused by the pressure applied on a noise barrier. For this initial study we used on purpose a very strong source in order to show the effects even with a material such as concrete. In the following, the ground is considered acoustically hard.

Let us consider a plane wave, a line and a point source.

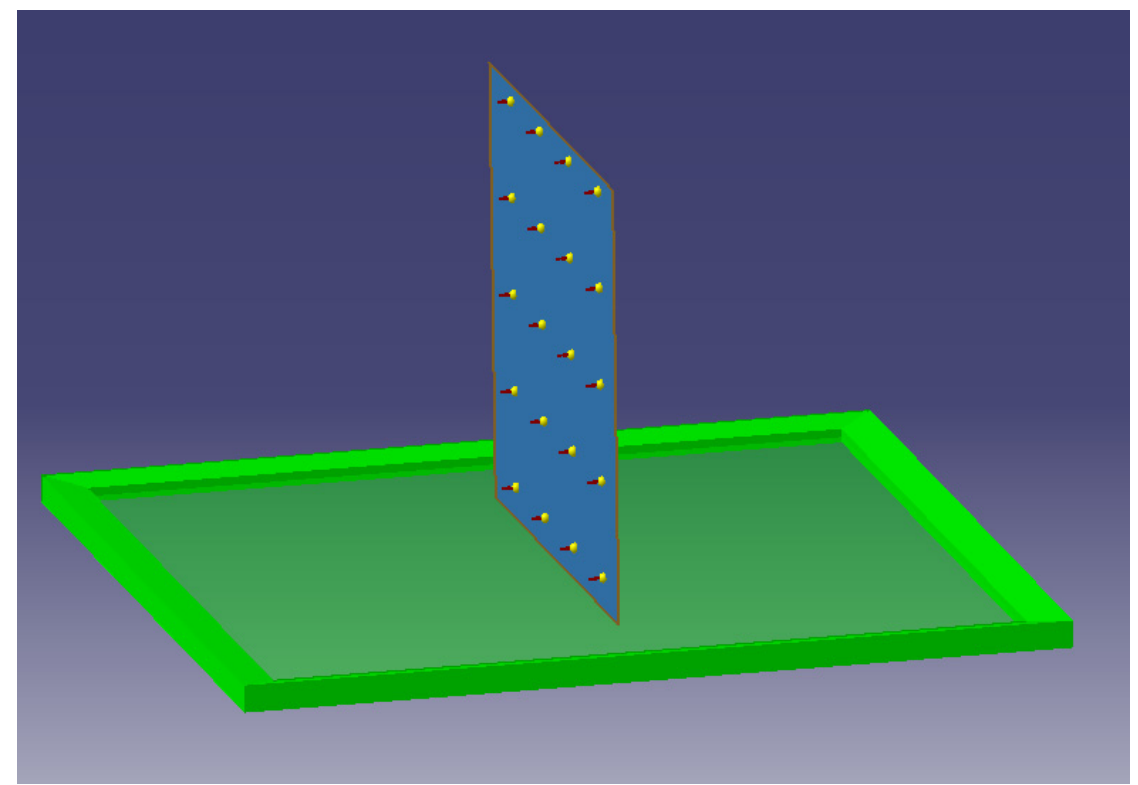

Figure 1: In our set-up the acoustical pressure is applied on a finite number of points. 
In the first case the pressure arriving on the barrier is always the same. On each point on the barrier the same force is applied. Simulations have been performed and give the results shown in Fig. 2.

We are observing here the effect of this kind of wave in the absence of the wave itself.

It is interesting to see that the effect is symmetrical and gives rise to an increase of the noise that is more marked at the ground level. Let us now observe the case of the point source.

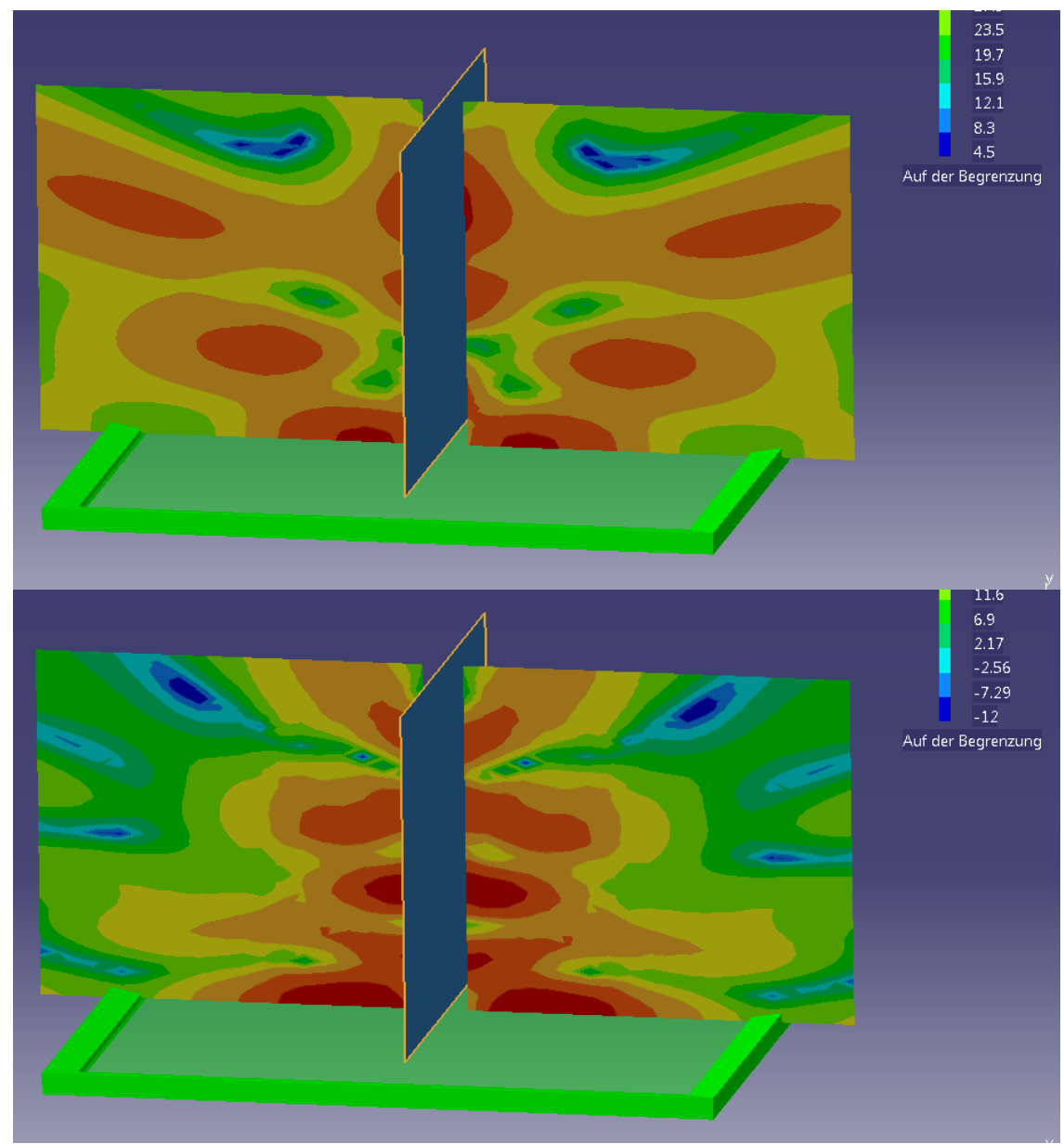

Figure 2: Secondary sound production under the effect of a plane wave $(160 \mathrm{~Hz}$ and $400 \mathrm{~Hz})$. 
We remember here that in the case of the point source the wave propagates in space so that the pressure follows this behaviour

$$
p \propto \frac{1}{r}
$$

where $r$ is the distance from the source, which allows us to calculate the pressure at the input points on the barrier. In our case the source is at $0.5 \mathrm{~m}$ away from the barrier, placed in the middle of it and $1 \mathrm{~m}$ from the ground.

Results are shown in the Fig. 3.

We proceed similarly in the case of a line source (cylindrical wave) considering that in this case the pressure dependence goes like

$$
p \propto \frac{1}{\sqrt{r}}
$$

The interesting thing that is common to all the shown figures is that the sound waves produced by the vibrating barrier tend to be focussed back in the position where the source is. In other words, the vibrational characteristics of the noise barrier for low frequencies amplify the effect of the source on the side of the barrier where the source is placed (in our case, on the left) and produce on the other side a kind of virtual source in a symmetrical position to the real source.

Our analysis was so far mainly of a qualitative nature and no information was given about the intensity of the secondary noise. We performed the same simulations with the acoustical source and obtained values that differ about 100 $\mathrm{dB}$ from the values shown here, which highly justify our choice of performing the simulations without source. Even if the secondary sound results in this case are inappreciable, this method will be used in the future for other materials and other shapes, where secondary sound will have a more important contribution.

Let us have a closer look at the results.

We put two microphone lines at two different heights, as shown in Fig. 5. The first line is on the ground, the second is at half height of the barrier $(2000 \mathrm{~m})$. In Fig. 6 the quantity transmission $\mathrm{T}$ is shown, defined as follows

$$
\begin{gathered}
T(d B)=10 \log _{10}(R) \\
R=\frac{1}{n} \sum_{i=1}^{n}\left(\frac{p_{i}}{\bar{p}_{0}}\right)^{2}
\end{gathered}
$$

where $p_{i}$ is the pressure at the nth position of the nth microphone and $p_{0}=20 \mu \mathrm{Pa}$ is the lower hearing limit. The different behaviour of the three sources can be seen. As expected, the plane wave has the biggest impact on the production of secondary sound waves. 


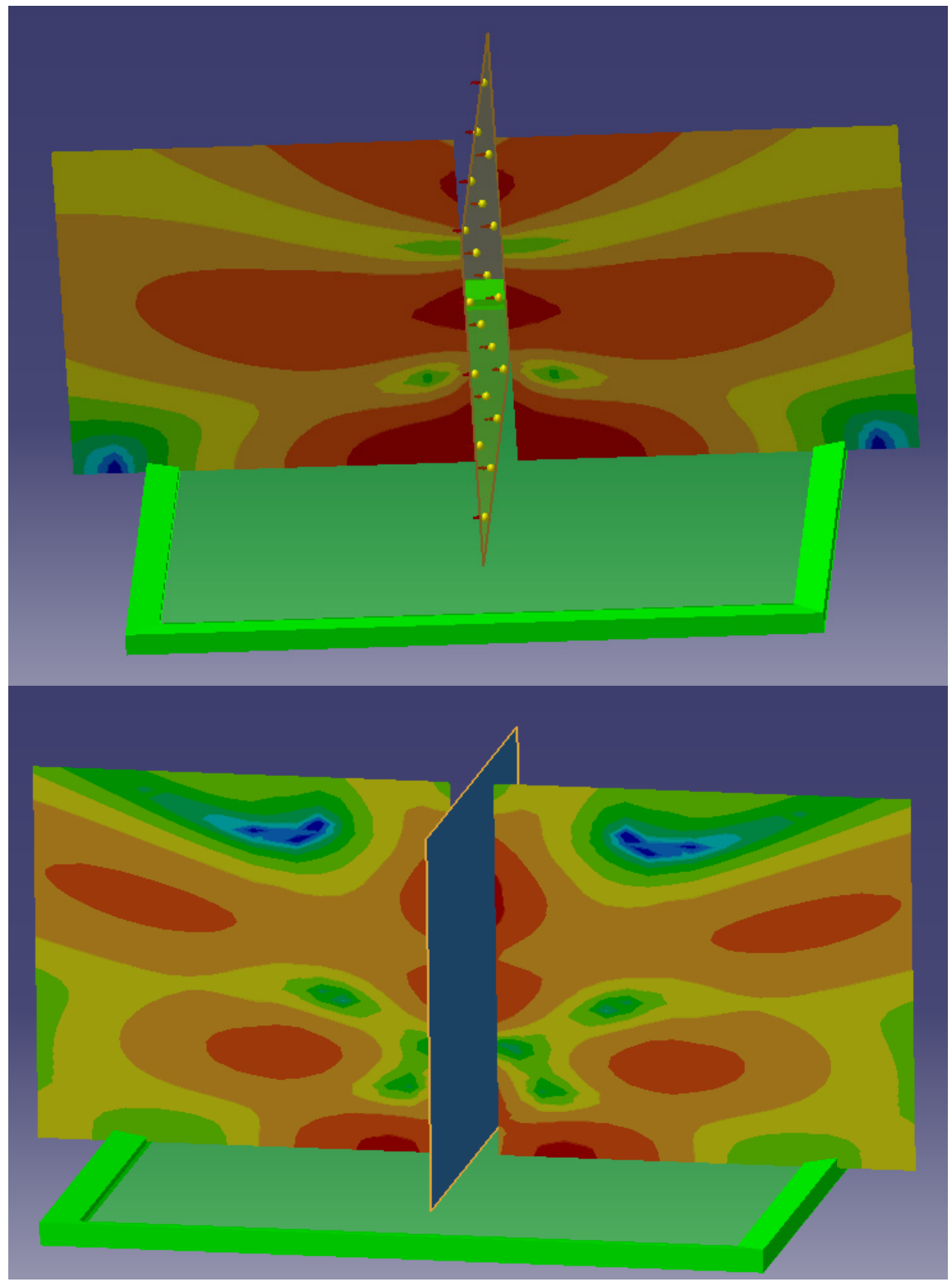

Figure 3: Secondary sound production under the effect of a point source $(160 \mathrm{~Hz}$ and $400 \mathrm{~Hz})$. 


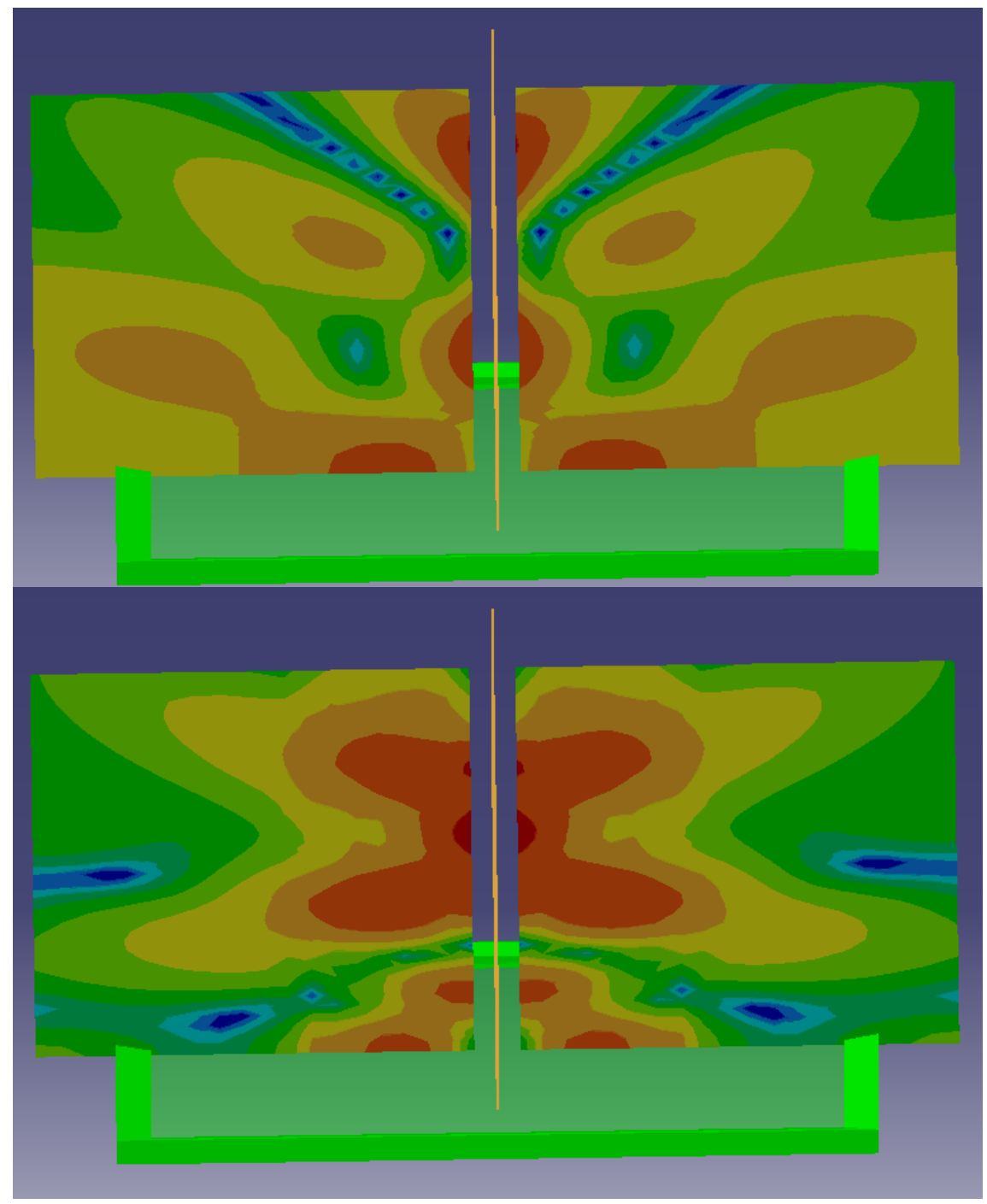

Figure 4: Secondary sound production under the effect of a cylindrical source $(160 \mathrm{~Hz}$ and $400 \mathrm{~Hz})$. 


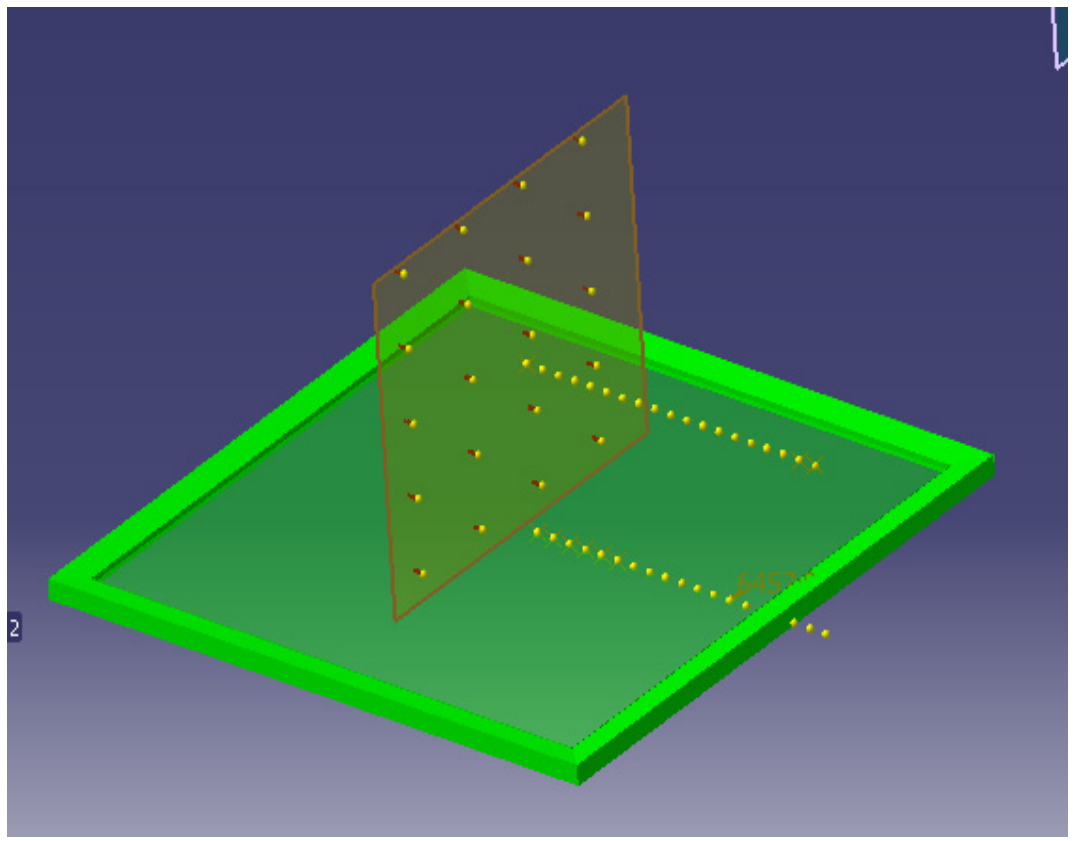

Figure 5: The two lines of microphones that have been used in the evaluation.

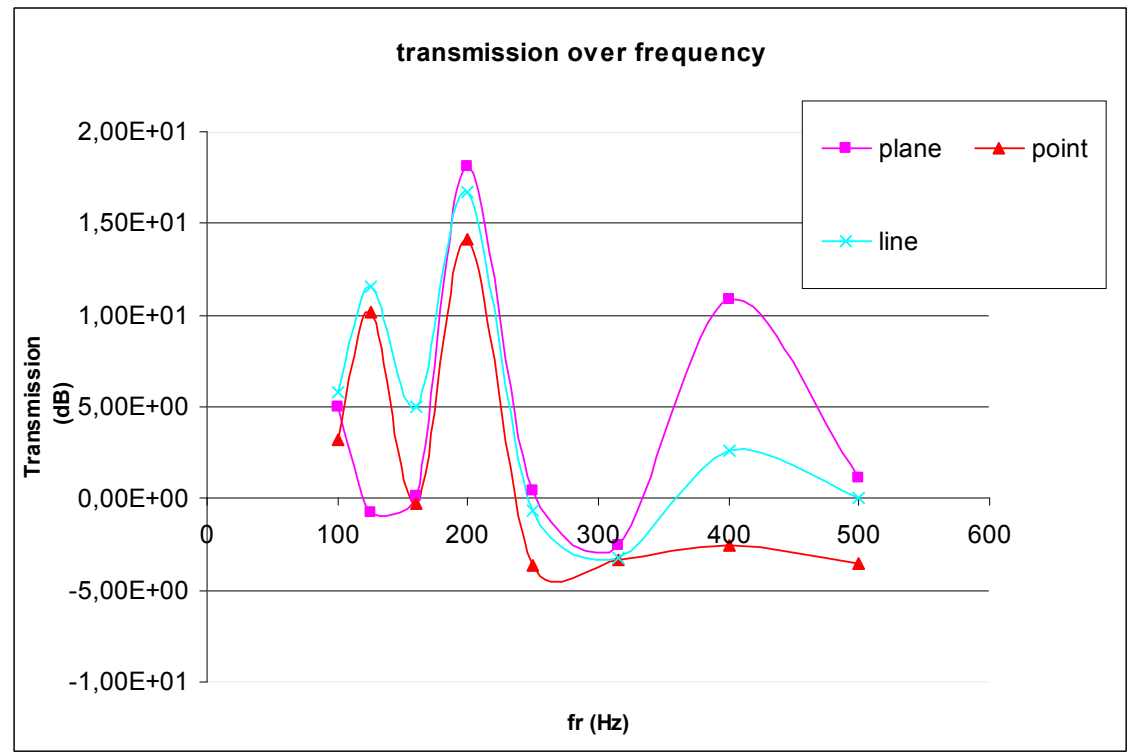

Figure 6: Transmission dependency on the frequency for the three different sources used. 


\section{Conclusions}

A new method has been used to perform vibro-acoustical simulations of finite noise barriers. This uses the integration of FE with BE methods, does not include the sound source but only uses the vibration of the noise barrier as a source.

The first results are encouraging and show an expected symmetry for the secondary sound. It also shows the building of an imaginary source in a symmetrical position with the real source.

\section{References}

[1] Seznec, R. Diffraction of sound around barriers: use of boundary elements technique. J. Sound Vibr., 73, 195-209 (1980)

[2] Gasparoni S., Haider M., Conter M., Wehr R., Breuss S., BEM simulations of noise barriers. Proceedings of the $39^{\text {th }}$ Internoise, SPA (2010)

[3] CEN, European Standard EN 1793-2 Road traffic noise reducing devices Test method for determining the acoustic performance - Part 1: Intrinsic characteristics of sound absorption, Belgium 1997

[4] CEN, European Standard EN 1793-2 Road traffic noise reducing devices Test method for determining the acoustic performance - Part 2: Intrinsic characteristics of airborne sound insulation, Belgium 1997 